\title{
Kournal of Biomedicine
}

2018; 3: 26-31. doi: 10.7150/jbm.23594

\section{Outcomes of Epidural Neuroplasty Utilizing Adhesiolysis by Means of Hydraulic and Mechanical force}

\author{
Isao Aoi ${ }^{*}$, Huan-Chieh Chen ${ }^{1,3^{*}}$, Tai-Ngar Lui ${ }^{1}$ and Tien-Jen Lin ${ }^{1,2,3 凶}$ \\ 1. Department of Neurosurgery, Wan Fang Hospital, Taipei Medical University, No. 111, Section 3, Xinglong Road, Wenshan District, Taipei City 116, Taiwan \\ 2. Graduate Institute of Injury Prevention and Control, Taipei Medical University, No.250, Wuxing Street, Xinyi District, Taipei City 110, Taiwan \\ 3. Graduate Institute of Sports Science, College of Exercise and Health Sciences, National Taiwan Sport University, Taoyuan City, Taiwan \\ *Isao Aoi and Huan-Chieh Chen made equal contribution to this article \\ $\triangle$ Corresponding author: Tien-Jen Lin; trlin1@hotmail.com
}

(C) Ivyspring International Publisher. This is an open access article distributed under the terms of the Creative Commons Attribution (CC BY-NC) license (https://creativecommons.org/licenses/by-nc/4.0/). See http://ivyspring.com/terms for full terms and conditions.

Received: 2017.11.22; Accepted: 2018.01.02; Published: 2018.02.16

\begin{abstract}
Background: Sciatica due to contained herniated discs can be managed with epidural neuroplasty which has emerged as a minimally invasive technique for the treatment of low back pain. Epiduroscopy also provides an alternative minimally invasive technique that offers diagnostic and therapeutic advantages. However, both procedures are not free of complications. The most commonly seen complications are related to the drugs and hypertonic fluid administered. The aim of this retrospective study is to evaluate the efficacy of epidural neuroplasty in the treatment of degenerative chronic low back pain and radiculopathy, and the prevention of complications by means of hydraulic and mechanical adhesiolysis with Cordis ${ }^{\circledR}$ catheter.

Methods: 112 patients treated by epidural neuroplasty were included in our retrospective, non-randomized case series. The outcomes and complications of epidural neuroplasty by hydraulic and mechanical adhesiolysis in patients with chronic low back pain or nerve irritation due to nerve root compression by a single-level, contained herniated disc were assessed. A blinded investigator assessed the patients before and at 3,6, and 12 months after treatment. Patients were asked to quantify their pain using the visual analog scale (VAS) and were also surveyed in regards to their pain medication usage. The Oswestry Disability Index (ODI) was quantified by third-party observers. Observed data at 3, 6, and 12 months after management were matched to baseline.

Results: The level of pain intensity and medication usage lessened considerably in the first three months after the management. An improvement in functional status was also observed in the first 3 months. Pain scores and medication use continued to increase and functional status continued to decline in our patients over the 12-month follow-up period. There were no procedure-related complications observed.

Conclusion: This retrospective study of mechanical adhesiolysis with Cordis ${ }^{\circledR}$ catheter demonstrated a statistically significant improvement in VAS pain scores, functional status and a reduction in medication intake. These findings suggest that epidural neuroplasty can be a safe and effective procedure for selected patients. To evaluate the long-term efficacy of this minimally invasive technique, further randomized, controlled studies are required.
\end{abstract}

Key words: Mechanical adhesiolysis, discectomy, disc herniation, low back pain, minimally invasive, caudal neuroplasty, epidurolysis

\section{Introduction}

Epidural neuroplasty (mechanical lysis of epidural adhesions), which was developed at Texas
Tech Health Sciences Pain Center in 1989, has emerged as an important interventional technique for 
the treatment of spinal or radicular pain that is refractory to conservative treatments. The procedure starts with placing an epidural catheter into the space between dura and the herniated disc or the scar tissue compromising the nerve root, then followed by simultaneous injections with local anesthetics, steroids, and normal saline. Improved pain scores and association with only minor complications were shown in the study conducted by Veihelmann et al [1]. Epiduroscopy is also a minimally invasive technique that offers diagnostic and therapeutic advantages in cases of chronic low back pain and radiculopathy. Saline instillation is used for distending the epidural space, but it cannot overcome fibrotic obstructions of the channel [2].

There are still few studies evaluating the safety and long-term efficacy of epidural neuroplasty at present. But epidural neuroplasty does successfully lessen pain in patients with contained herniated discs in six months [3, 4], however, epidural neuroplasty is not free of complications. Some complications are related to the procedure itself or the drugs administered. The aim of this retrospective study was to evaluate the efficacy of epidural neuroplasty in the treatment of degenerative chronic low back pain, and the prevention of complications by purely mechanical adhesiolysis with Cordis ${ }^{\circledR}$ catheter. Outcomes of 112 non-randomized patients treated with purely mechanical adhesiolysis were evaluated relatively to the following considerations: gradual reduction of pain intensity, improvement in functional activity, reduction of analgesic use longitudinally over 1 year and procedure-related complication in treated patients.

\section{Materials and methods}

\section{Patient Selection}

This retrospective study was conducted in the Department of Neurosurgery, Wan-Fang Medical Center, Taipei Medical University, Taipei, Taiwan. A total of 112 patients with a history of chronic low back pain and sciatica managed with epidural neuroplasty between January 2010 and March 2014.

The patients enrolled were those who suffered leg and back pain after failed conservative therapy for 9 weeks. All patients received MRI study with evident contained disc protrusion with a disk height $\geqq 50 \%$. Provocative discography was conducted on all subjects. A concordant pain response accompanying the demonstration of contained disc by performing the discography, with a painless response at an adjacent spinal level, became the foundation for patient enrollment in our study. Patients with lesion disc height $<50 \%$, complete annular disruption revealed by discography, more than one symptomatic disc levels, a history of open disc surgery at treatment levels and moderate to severe spinal stenosis were excluded. Patients' data such as age, gender, location of pain, level of procedure performed, duration of the procedure, pre- and post-procedural visual analog scale (VAS) pain scores, Oswestry Disability Index (ODI), medication intake and procedure-related complication were carefully valued.

\section{Procedures}

The procedure was performed on an inpatient basis in the operating room. Percutaneous epidural neuroplasty was performed under monitored anesthesia care in the usual sterile fashion. The patient was placed in the prone position with sterile drapes in position and the procedure was performed in a caudal approach under fluoroscopy. After local anesthetic infiltration of the skin on the top of the gluteal fold, a 14- gauge needle was inserted via the sacral hiatus on the side contra-lateral to the alleged pain generator. Confirmation of ventral placement of the catheter was done with the help of a lateral fluoroscopic view. With confirmation of the needle in the epidural space, we injected $10 \mathrm{~mL}$ of iohexol (Omnipaque ${ }^{\mathrm{TM}}$ ) after negative aspiration and visualized spread of the contrast medium (epidurogram). The steel needle was withdrawn and leaving the sheath for the introduction of the Cordis ${ }^{\circledR}$ catheter into the epidural space. If a filling defect corresponding to the pain generator was present, we made the way with the Cordis $^{\circledR}$ catheter towards the filling defect (Figure 1). To open up the adhered epidural space we infused with 10 to $50 \mathrm{ml}$ of normal saline as the first step. Following this phase, mechanical dissection of the connective structures was performed. The procedure started with a slow advancement of the catheter, using the guide wire to further open up adhered epidural space in order to reach the pathological area (Figure 2a and $2 b$ ). The maneuvers can be repeated for 2-3 times until the guidewire can be advanced through the adhered space freely. A supplementary 2 to $3 \mathrm{ml}$ of iohexol was injected to visualize the opening of the scarred or adhered area within the epidural space. After injection of $4 \mathrm{ml}$ of $40 \mathrm{mg} / \mathrm{ml}$ methylprednisolone, both the needle sheath and catheter were withdrawn.

\section{Outcome Measures}

Patients were evaluated preoperatively and at 3 , 6, and 12 months post-operatively. Data collected included quantification of pain, pain medication 
intake, and changes in performance levels of daily living activities.

\section{Quantification of pain}

At each evaluation, patients were asked to quantify their overall pain using a VAS pain score ranging from 0 to 10 .

\section{Functional status}

The Oswestry Disability Index is applied in this study to measure the outcome and assess the disabling effects of lumbar spinal disorders.

Analgesic intake

Patients were surveyed in regard to their use of analgesics. If a patient reported with complete cessation of analgesics intake or a daily reduction of $50 \%$ or more is deemed as reduced analgesic use.

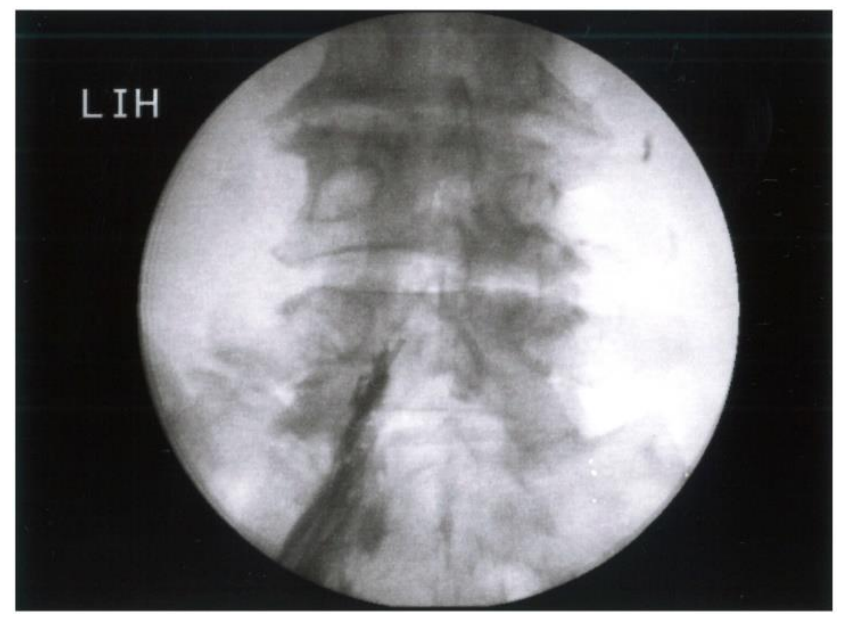

Figure 1. Anteroposterior view of correct placement of the catheter with confirmation by dye spread. Note that a filling defect was observed between L4/5 disc space.

\section{Statistical Analyses}

Outcome measure data at baseline was compared longitudinally to evaluations taken at 3,6, and 12 months post-treatment. Wilcoxon matchedpairs signed-ranks test was used for VAS pain score analysis. Furthermore, Fisher's exact test and the Wilcoxon ranks sum test were used to identify factors that were significantly associated with changes in other variables. All statistical tests were two-sided, and $\mathrm{P}$ values $<0.05$ were considered to be statistically significant.

\section{Results}

\section{Patient Demographics and Baseline Data}

Analysis of outcomes of utilizing epidural neuroplasty was performed on 112 patients. There were 60 men and 52 women ( $47.71 \pm 9.89$ years of age). All patients were given written consent, and the study fulfilled the criteria of the ethics committee of Wan Fang Hospital.

Axial back pain was reported by $21(18.75 \%)$ of the patients, while $91(81.25 \%)$ reported back and leg pain. Most patients $(80.36 \%)$ had pain for over 12-months duration. Mean procedure duration was 14 minutes for the epidural neuroplasty treatment.

\section{Post-Surgical Outcomes}

There were no complications related to the procedures. All patients were successfully treated without any significant complications during the procedure. Hospital stays ranged from 2 to 4 days, with an average of 2.07 days. 112 patients had one disc treated; a total of 112 procedures were performed. The mean follow-up period was $12 \pm 1.9$ months. Four candidates were lost to 1-year follow-up due to inadvertent discectomies.

Mean VAS was reduced from a pre-procedure score of 7.69 to a post-procedure score of 3.92 at 3 months, 5.30 at 6 months and 5.55 at 12 months in the epidural neuroplasty group (Figure 3 ). Overall patient satisfaction was $60.7 \%$ (68 in 112 patients) at 3 months, $56.3 \%$ (63 in 112 patients) at 6 months, and $55.6 \%$ (60 in 108 patients) at the latest follow-up. Twelve months of follow-up showed that 36 patients were completely satisfied with the treatment (mean VAS score 0-2) and had complete resolution of symptoms, 40 patients obtained a good result (mean score 3-4), 20 patients received little benefit (mean score 5-7), and in 16 patients results were completely unsatisfactory (mean score 8-9). VAS pain scores significantly decreased (VAS reduction more than 4 in scale) in $74.1 \%$ (83 in 112), $62.5 \%$ (70 in 112), and 60.2 $\%$ (65 in 108) of patients at 3, 6, and 12 months, respectively with a mean decrease of $3.768(\mathrm{P}<0.001)$, and then increase $1.375(\mathrm{P}<0.001)$, and $0.250(\mathrm{P}=$ $0.50)$ noted, respectively (Table 1 ).

Table 1. Change in post-treatment VAS pain scores over one year.

\begin{tabular}{llll}
\hline Follow Up & 3 months & 6 months & 12 months \\
\hline Mean & -3.768 & -2.393 & -2.143 \\
Median & -3 & -3 & -2 \\
Range & $(-8.0,0)$ & $(-8.0,0)$ & $(-8.0,0)$ \\
Signed-ranks test P value & $<0.001$ & $<0.001$ & 0.5 \\
\hline
\end{tabular}

ODI had decreased in treated patients from a pre-procedure score of $22.1 \pm 8.5$ to a post-procedure score of $18.9 \pm 7.5$ at 3 months, $23.1 \pm 8.1$ at six months and $23.4 \pm 8.9$ at 12 months. 
Analgesic consumption was stopped or reduced in 91 patients at 3 months. The percentage of patients reporting a reduction in analgesics intake was $81.3 \%$ (91 in 112) at 3 months ( $P<0.0001), 76.8 \%$ (86 in 112) at 6 months $(\mathrm{P}<0.0001)$, and $69.4 \%(75$ in 108) at 12 months $(\mathrm{P}<0.0001)$.

\section{Failure of Treatment and Complications}

We did not find any significant factors related to failure of treatment in our study. In addition, there were no intra-operative or post-operative complications associated with epidural neuroplasty.

\section{Discussion}

\section{Pathological basis of discogenic pain}

Discogenic pain is the result of a complex interplay of biomechanical and biochemical processes. Not only the nerve root is capable of pain sensation, innervation of the ventral epidural space is extensive. Histopathological studies have demonstrated extensive sinuvertebral nerve and sympathetic innervation over this confined space [5]. These structures may become highly sensitized, resulting in chronic low back pain.

Intervertebral disc-related pain can be caused by structural abnormalities, such as degeneration or herniation. First to create widespread interest in the disc as a source of pain in the American literature were Mixter and Barr [6] with their description of the herniated nucleus pulposus. In a review of the literature, Benzon [7] pointed out that abnormalities of the intervertebral disc include degeneration, bulging, and herniation. Bulging of the disc distends the posterior longitudinal ligament and causes localized back pain. If bulging of the disc increases, pressure may be exerted on the adjacent nerve roots, producing radicular pain.

Chemical irritation is also a major contributing factor in the origin of pain. Radicular pain can occur without disc herniation. Internal disc disruption is a condition in which the internal architecture of the disc is disrupted but its external surface remains essentially normal [8]. Stolker [9] believed that mechanical factors are not the only causative factors of radicular pain. Nerve roots may be exposed to chemical irritant substances from degenerated intervertebral discs or facet joints, which can generate pain. Mixter and Ayers [10] also demonstrated the same conclusion. The leaked nucleus pulposus has been identified as a source of chemicals which produce annoyance [11]. Substances that produce inflammation include lactic acid, glycoprotein, cytokines, and histamine. In addition, it has been theorized that material from the nucleus pulposus might act as a foreign protein and trigger an autoimmune reaction.

The identification of fibrosis as the origin of low back pain has never been debated. In patients who had undergone prior surgeries, there was always some degree of perineural fibrosis. Although scar tissue itself was never tender, the nerve root was frequently very sensitive. Kuslich et al. [12] suggested that the presence of scar tissue compounded pain associated with the nerve root by fixing it in one position and thus increasing the susceptibility of the nerve root to tension or compression.

\section{Complications of epidural neuroplasty using hypertonic saline}

Unintended subarachnoid or subdural puncture with injection of hypertonic saline or local anesthetic is one of the major complications of epidural neuroplasty. Manchikanti et al [13-16] reported one subarachnoid block in 75 studied patients [13]; one suspicious infection in 30 studied cases [14]; 5 subarachnoid blockade, one serious infection and 4 suspicious infection in a retrospective evaluation of 232 patients [15]; 4 subarachnoid puncture, one infection and 8 suspicious infection in 178 procedures [16]. There were no other serious complications reported such as arachnoiditis, paralysis, weakness or bladder disturbances in their studies, but minor complications like rash or itching were mentioned. Veihelmann et al [17] reported 15 cases of transient sensory deficit, 2 subarachnoid catheters and one sheared catheter in 47 subjects. Gerdesmeyer et al [18] reported 2 partially sheared catheters and one infection in 61 treated patients. Talu et al [19] reported $3(1.2 \%)$ epidural abscesses and one severe headache in 250 patients. Perkins et al [20] described one patient who underwent percutaneous adhesiolysis to treat persistent back and leg pain after two previous lumbar surgeries with a retained, sheared catheter. Wagner et al [21] reported a case of severe meningitis after percutaneous adhesiolysis. Ho et al [22] described a patient who developed acute monoplegia immediately after the procedure. They concluded that large volumes of fluid injected during epidural neuroplasty could have caused transient nerve injury from compression within loculated epidural compartments. In a review of literature by Gill et al [23], twelve cases of visual impairment following epidural fluid injection have been reported. The common finding was retinal hemorrhage, and bolus injection of fluid was considered to be the precipitating event. 


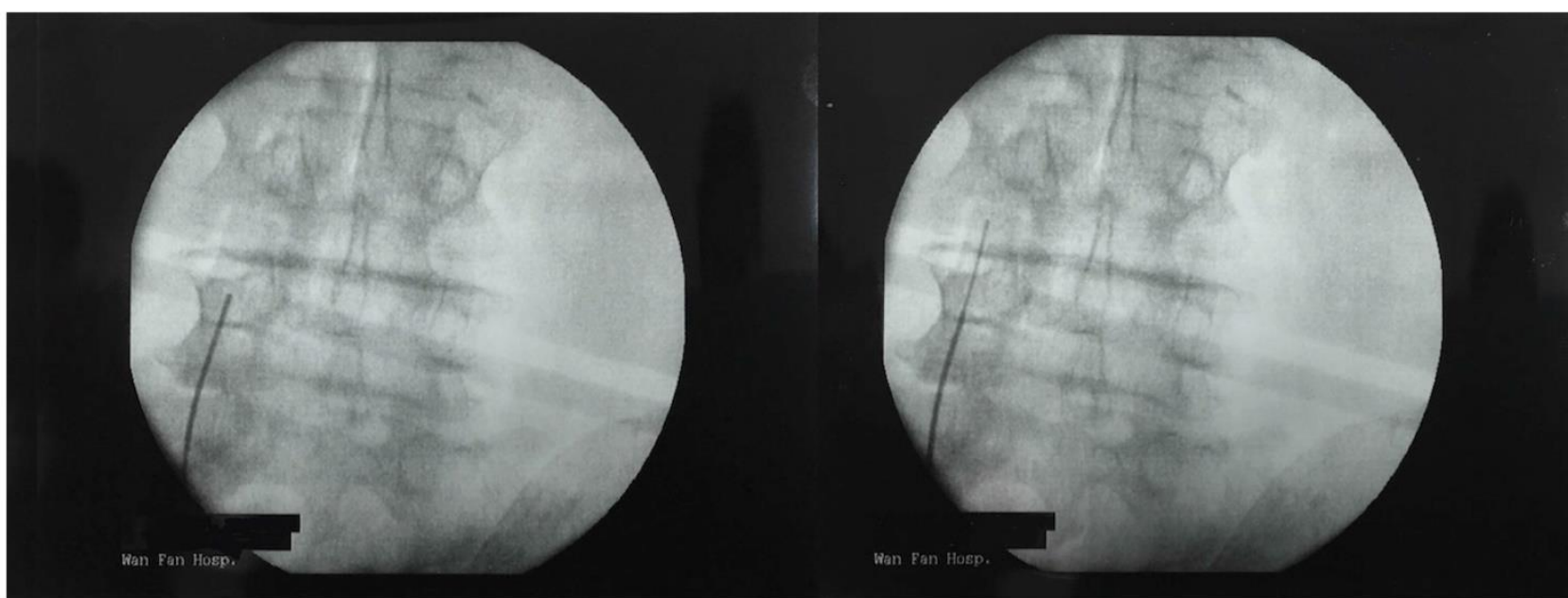

Figure 2. To further open up adhered epidural space in order to reach the pathological area, we advanced the catheter with the help of the guide wire (right). The maneuvers can be repeated for 2-3 times until the guide wire can be advanced through the adhered space. In this phase, mechanical dissection of the connective structures was performed.

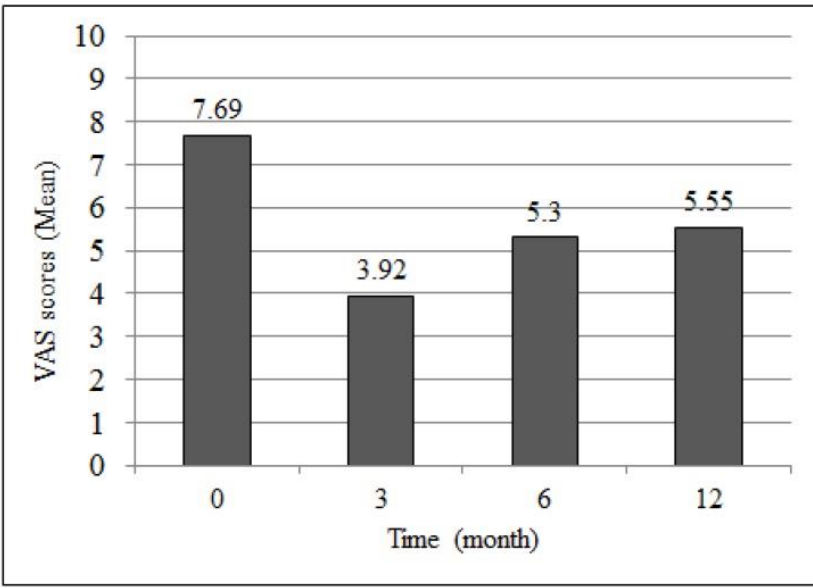

Figure 3. Mean pain VAS scores post-procedure over one year.

Shah and Heavner [24] described the visual and fluoroscopic clues indicating subarachnoid and subdural placement of the catheter. Subdural spread could be recognized due to the rapid rise of the contrast column and the characteristic pattern of appearance: increased opacity, flat dural margin, irregular arachnoid margin, and the absence of lateral spread along the spinal nerves. Subarachnoid placement could be confirmed by direct visualization of the nerve roots and associated blood vessels, a myelographic dye pattern and the aspiration of cerebrospinal fluid.

\section{The advantages of epidural neuroplasty by means of hydraulic and mechanical force}

Our goal of treating discogenic low back pain and leg pain is primarily based on the mechanisms underlying postulated disc remodeling treatment, which results in reduction in levels of inflammatory mediators released into the epidural space and release of "trapped" nerve root by mechanical adhesiolysis. Pre-procedural epidurography provided substantial importance for assurance that no extravasations of contrast material were present and concordant level of the origin of pain was the target before proceeding with the intervention thus allowing the result of the treatment to be optimum. Compared with other minimally invasive procedures, our treatment has minimal damages to the surrounding tissue due to less volume of epidural fluid injected and mechanical disruption of adhesion bands without drug administrations, leading to less intra-operative and postoperative pain, allowing for quick rehabilitation. The advantage of this procedure is that the Cordis ${ }^{\circledR}$ catheter has been introduced into epidural space via the plastic 14-gauge sheath permitting less possibility of catheter shearing during the maneuver. On the other hand, the catheter has been withdrawn immediately after the procedure thus minimizing the chance of developing epidural abscess.

\section{Limitations of this study}

Although other studies have also shown an overall reduction in pain scores following percutaneous epidural neuroplasty, these studies have only shown a general decline in pain relief over time. Interestingly, pain scores and medication use continued to increase and functional status continued to decline in our patients over the 12-month follow-up period compared to other previous investigations. Nevertheless, 76 in 112 patients still showed either excellent or good result 12 months after the procedure. 
Our study has several limitations. The retrospective nature of this study is a disadvantage. The sample size is relative small and the results may not be applicable to all patient populations. Despite these inherent weaknesses, our study does help to provide a preliminary outline for the planning of future prospective, randomized, controlled studies to justify whether epidural neuroplasty by means of hydraulic and mechanical force is superior to other treatment or physiotherapy.

\section{Conclusion}

This retrospective study of treatment with epidural neuroplasty demonstrated a statistically significant improvement in VAS pain scores, functional status and a reduction in medication intake in a group of patients with radicular or axial low back pain who had failed conservative treatment. The findings suggest that epidural neuroplasty by means of hydraulic and mechanical force can be a safe and effective procedure for selected patients. While we believe the risk of this procedure to be small, it should be taken under advisement while evaluating patient eligibility. Further prospective, randomized, controlled studies are needed to evaluate the long-term efficacy of this minimally invasive technique.

\section{Competing Interests}

The authors have declared that no competing interest exists.

\section{References}

1. Veihelmann A, Devens C, Trouillier H, Birkenmaier C, Gerdesmeyer L, Refior HJ. "Epidural neuroplasty versus physiotherapy to relieve pain in patients with sciatica: a prospective randomized blinded clinical trial". I Orthop Sci. 2006; 11( 4): 365-369.

2. M. Avellanal and G. Diaz-Reganon, "Interlaminar approach for epiduroscopy in patients with failed back surgery syndrome". Br J Anaesth. 2008; 101(2): 244-249.

3. Manchikanti L, Pampati V, Fellows B, Rivera J, Beyer CD, and Damron KS. "Role of one day epidural adhesiolysis in management of chronic low back pain: a randomized clinical trial". Pain Physician. 2001; 4(2):153-166.
4. Heavner JE, Racz GB, and Raj P. "Percutaneous epidural neuroplasty: prospective evaluation of $0.9 \% \mathrm{NaCl}$ versus $10 \% \mathrm{NaCl}$ with or without hyaluronidase". Reg Anesth Pain Med. 1999; 24(3):202-207.

5. Cautico W, Parker JC, Pappert E, Pilsl S. "An anatomical and clinical investigation of spinal meningeal nerves". Acta Neurochir (Wien). 1988; 90(3): 139-143.

6. Mixter WJ, Barr JS. "Rupture of the intervertebral disc with involvement of the spinal canal". N Eng J Med. 1934; 211(5):210-215.

7. Benzon HT. "Epidural steroid injections for low back pain and lumbosacral radiculopathy". Pain. 1986; 24(3): 277-295.

8. Crock HV. "Isolated lumbar disc resorption as a cause of nerve root canal stenosis". Clin Orthop Relat Res. 1976; 115(2):109-115.

9. Stolker RJ, Vervest ACM, Groen GJ. "The management of chronic spinal pain by blockades: A review". Pain.1994; 58(1): 1-20.

10. Mixter WJ, and Ayers JB. "Herniation or rupture of the intervertebral disc into the spinal canal - report of thirty-four cases". N Engl J Med. 1935; 213(9): 385-393,.

11. Racz GB, Noe C, Heavner JE. “Selective spinal injections for lower back pain". Curr Rev Pain. 1999; 3(5):333-341.

12. Kuslich SD, Ulstrom CL, Michael CJ. "The tissue origin of low back pain and sciatica: A report of pain response to tissue stimulation during operation on the lumbar spine using local anesthesia". Orthop Clin North Am. 1991; 22(2):181-187.

13. Manchikanti L, Rivera J, Pampati V, Damron KS, McManus CD, Brandon DE, and Wilson SR, "One day lumbar epidural adhesiolysis and hypertonic saline neurolysis in treatment of chronic low back pain: A randomized double blind trial". Pain Physician. 2004; 7(2): 177-186.

14. Manchikanti L, Pampati V, Fellows B, Rivera JJ, Beyer CD, and Damron KS, "Role of one day epidural adhesiolysis in management of chronic low back pain: A randomized clinical trial". Pain Physician. 2001; 4(2):153-166.

15. Manchikanti L, Pakanati R, Bakhit CE, and Pampati V, "Role of adhesiolysis and hypertonic saline neurolysis in management of low back pain: evaluation of modification of the Racz protocol". Pain Digest. 1999; 9(9): 91-96.

16. Manchikanti L, Pampati V, Bakhit CE, Pakanati RR. "Non-endoscopic and endoscopic adhesiolysis in post-lumbar laminectomy syndrome: a one-year outcome study and cost effectiveness analysis". Pain Physician. 1999; 2(3): 52-58.

17. Veihelmann A, Devens C, Trouiller H, Birkenmaier C, Gerdesmeyer L, and Refior HJ, "Epidural neuroplasty versus physiotherapy to relieve pain in patients with sciatica: a prospective randomized blinded clinical trial". I Orthop Science. 2006; 11(4):365-369.

18. Gerdesmeyer L, Lampe R, Veihelmann A, Burgkart R, Gobel M, Gollwitzer H, and Wagner $\mathrm{K}$, "Chronic radiculopathy. Use of minimally invasive percutaneous epidural neurolysis according to Racz". Schmerz. 2005; 19(4): 285-295.

19. Talu GK and Erdine S, “Complications of epidural neuroplasty: a retrospective evaluation". Neuromodulation. 2003; 6( 4): 237-347.

20. Perkins WJ, Davis DH, Huntoon MA, and Horlocker TT, "A retained Racz catheter fragment after epidural neurolysis: implications during magnetic resonance imaging". Anesth Analg. 2003; 96(6): 1717-1719.

21. Wagner KJ, Sprenger T, Pecho C, Kochs EF, Tölle TR, Berthele A, Gerdesmeyer L. "Risks and complications of epidural neurolysis - a review with case report". Anasthesiol Intensmed Notfallmed Schmerzther. 2006; 41(4): 213-222.

22. Ho KY and Manghnani $P$, "Acute monoplegia after lysis of epidural adhesions: a case report". Pain Pract. 2008; 8(5):404-407.

23. Gill JB, Heavner JE. "Visual impairment following epidural fluid injections and epiduroscopy: a review". Pain Med. 2005; 6(5): 367-374.

24. Shah RV and Heavner JE, "Recognition of the subarachnoid and subdural compartments during epiduroscopy: two cases". Pain Pract. 2003; 3(4): 321-325. 